\title{
Creatinine-based definitions: from baseline creatinine to serum creatinine adjustment in intensive care
}

\author{
Silvia De Rosa*, Sara Samoni and Claudio Ronco
}

\begin{abstract}
This article is one of ten reviews selected from the Annual Update in Intensive Care and Emergency medicine 2016. Other selected articles can be found online at http://www.biomedcentral.com/collections/ annualupdate2016. Further information about the Annual Update in Intensive Care and Emergency Medicine is available from http://www.springer.com/ series/8901.
\end{abstract}

\section{Background}

There is still controversy in the literature regarding the diagnosis of acute kidney injury (AKI), despite the latest definition provided by the Kidney Disease - Improving Global Outcomes (KDIGO) working group created in 2012. Standardized definitions of AKI, using changes in serum creatinine ( $\mathrm{SCr}$ ) and urine output, have enabled a better evaluation of the epidemiology of intensive care unit (ICU)-acquired AKI, including long-term outcomes in patients who have experienced AKI [1]. Very few studies have considered the correlation of baseline renal function with increased creatinine concentrations during the episode of AKI [2]. Although the AKIN (Acute Kidney Injury Network), KDIGO and ERBP (European Renal Best Practice) groups have contributed to standardizing the diagnosis and staging of AKI, the lack of a joint approach to baseline serum creatinine (bSCr) concentration has influenced the creatinine-based AKI definitions. This concept is complicated by other factors, such as: (i) the absence of standardized laboratory methods for quantification of SCr; (ii) arbitrary cut-offs for $\mathrm{SCr}$ in the diagnosis of $\mathrm{AKI}$, which compromises the validity of studies because of the different classifications used [3, 4]; (iii) the presence of pre-existing undiagnosed

\footnotetext{
* Correspondence: derosa.silvia@ymail.com

San Bortolo Hospital, International Renal Research Institute, 36100 Vicenza, Italy
}

chronic kidney disease (CKD) in a large part of the population; (iv) unknown baseline status of renal function in patients affected by AKI. All these reasons participate to the inability to define a 'universal bSCr'. The absence of a shared approach has resulted in variability among centers, with different interpretations of the $\mathrm{SCr}$ value for the definition and classification of AKI. Moreover, in the ICU setting, fluid overload and muscle wasting can delay recognition of AKI by affecting the true value of the SCr. Recent studies have demonstrated the effect of fluid balance on SCr: a positive fluid balance can dilute $\mathrm{SCr}$, decreasing the ability to identify the development of AKI [5-8]. In terms of muscle wasting, there are no data available regarding its effect on $\mathrm{SCr}$ and, moreover, ICU-acquired myopathy is rarely recognized because of insufficient diagnostic criteria or methodological limitations $[9,10]$. Despite these limits, $\mathrm{SCr}$ is still considered the standard for assessing acute changes in renal function. The present review details the existing evidence related to the importance of bSCr in ICU patients, describing in particular the limits of the non-shared approach. We also attempt to highlight the effects of fluid balance and muscle wasting on $\mathrm{SCr}$ based on recent evidence.

\section{Estimating baseline serum creatinine}

In 2004, recommendations drawn up by the Acute Dialysis Quality Initiative (ADQI) working group defined a value of estimated glomerular filtration rate (eGFR) [75$\left.100 \mathrm{ml} / \mathrm{min} / 1.73 \mathrm{~m}^{2}\right]$, that was useful for defining the value of bSCr using the Modification of Diet in Renal Disease (MDRD) equation (back-estimation) in patients with unknown baseline renal function and in the absence of prior renal disease [11]. The back-estimation formula is:

$$
\begin{aligned}
\text { Serum creatinine }= & \left(75 /\left[186 \times\left(\text { age }^{-0.203}\right) \times(0.742 \text { if female })\right.\right. \\
& \times(1.21 \text { if black })])^{-0.887}
\end{aligned}
$$

Unfortunately, in patients with suspected CKD, the back-estimation formula overestimates the incidence of 
AKI and should not be used [3]. This method, although used in several epidemiological studies, lacks scientific validation $[12,13]$. Two other methods are used to determine the bSCr: the first $\mathrm{SCr}$ measured at admission; and the nadir (the lowest measured value) of $\mathrm{SCr}$ during the first three days of the ICU stay. RIFLE and KDIGO suggest using the back-estimation formula, whereas AKIN recommends using the first measurement of $\mathrm{SCr}[3,14,15]$. ERBP and NICE (The National Institute for Health and Care Excellence) guidelines suggest using the admission $\mathrm{SCr}$ value because of the correlation of this value with patient outcomes, such as mortality and need for renal replacement therapy (RRT). In a large study, Broce et al. estimated the baseline renal function in patients with AKI using the nadir $\mathrm{SCr}$ measured in the first three days of hospitalization. Hospital-acquired AKI was defined according to the difference between the nadir and the subsequent peak SCr. Different thresholds of nadir-to-peak $\mathrm{SCr}$ were independently associated with increased in-hospital mortality. In addition, the time lag between the nadir $\mathrm{SCr}$ and the peak SCr was seven days [16]. Several authors have demonstrated an over-estimation of $50 \%$ using MDRD back-estimation in different patient populations [3]. In a cohort study, Siew et al. estimated bSCr in a population of almost 5000 hospitalized patients using the three different methods [15]. The MDRD equation and nadir $\mathrm{SCr}$ overestimated the incidence of AKI by at least $50 \%$ and the admission SCr underestimated it by $46 \%$ $[4,15]$. The use of admission SCr had the lowest sensitivity for diagnosis of hospital-acquired AKI [3] and missed the diagnosis of community-acquired AKI. For this reason, admission $\mathrm{SCr}$ should be used with caution, especially in AKI stage 1 [16]. Moreover, in addition to these three estimation methods, some authors have also considered the pre-admission SCr when it is available, but few have defined the timing of this measurement. For example, Matheny et al. proposed a time-period of a maximum of 365 days and minimum of 7 days from the moment of hospital admission [14, 17]. Unfortunately, pre-admission $\mathrm{SCr}$ is often not available [1]. Recent data support a bidirectional relationship between AKI and CKD. Some patients with AKI can develop CKD because their renal function does not return to normal. For this reason, it is important to identify patients at risk of developing CKD or at risk of progressing to end-stage renal disease (ESRD). In order to improve the use of $\mathrm{SCr}$ for diagnosing AKI and CKD, biomarkers of renal function could be helpful.

\section{Baseline serum creatinine in cardiac surgery and pediatrics Cardiac surgery}

The development of AKI in the cardiac surgery setting is complex: exposure to nephrotoxins (e.g., radio-contrast from cardiac catheterization, antibiotics), preexisting conditions (e.g., diabetes mellitus, CKD), and exposure to intraoperative events (e.g., duration of cardiopulmonary bypass $[\mathrm{CPB}]$, hypotension) can all influence the risk of AKI and patient outcomes [18]. Elevated preoperative $\mathrm{SCr}$ is an independent risk factor for morbidity and mortality after cardiac surgery [19]. Small postoperative increases in $\mathrm{SCr}$ of $20-25 \%$ from preoperative baseline are associated with adverse outcomes [20-22]. However, SCr values are poor predictors of AKI in the early period after cardiac surgery [23]. In this setting, creatinine-based AKI definitions are not easy to use and very often the $\mathrm{bSCr}$ value used is the preadmission or admission SCr. Meersch et al. [24] defined the bSCr as the median of all values available from six months to one day prior to cardiac surgery; if this was not possible, the $\mathrm{SCr}$ value determined one day before cardiac surgery was used. Renal recovery from AKI was defined as an $\mathrm{SCr}$ value at hospital discharge equal to or less than the bSCr [23]. Other recent studies have shown that use of the bSCr from the MDRD equation (back-estimation) overestimated the incidence of AKI in patients undergoing cardiac surgery $[15,25]$. One possible explanation could be that in these special populations, the average patient age is high and there is a large prevalence of vascular disease and consequently more CKD than in the general population. When these classes of patients are excluded, the overestimation decreases. Indeed, as explained earlier, the MDRD equation (back-estimation) can be used in patients with normal or nearly normal premorbid GFR, but it overestimates AKI in populations with an increased prevalence of mild CKD.

\section{Pediatrics}

In children, the bSCr is often unknown and little information is available concerning outcomes after AKI. Variations in estimating bSCr have led to clinically significant differences in ascertainment of AKI incidence. Zappitelli et al. [26] showed that, depending on which bSCr measurement was used, AKI patterns, incidence and outcome associations differed substantially. The authors suggested that change in estimated creatinine clearance $(\mathrm{eCrCl})$ be used to define $\mathrm{AKI}$ and, when $\mathrm{bSCr}$ is unknown, the Schwartz $\mathrm{eCrCl}$ of $120 \mathrm{ml} / \mathrm{min}$ per $1.73 \mathrm{~m}^{2}$ should be used to estimate baseline renal function. In another study [27], the authors showed that bSCr was unknown in over $50 \%$ of the study population. They used center-specific age- and sex-based normative values in all patients versus only in those with an unknown bSCr. Results showed that normative estimated bSCr might be a reasonable alternative when 'true' bSCr data are unavailable, which is particularly likely to occur in population-based studies. In addition, they showed that in many children who develop AKI, SCr had not returned to $\mathrm{bSCr}$ values at the last pediatric $\mathrm{ICU} \mathrm{SCr}$ 
assessment and that values were less likely to return to bSCr values in those with worse AKI. These children could be at risk of long-term renal disease [25]. In a pediatric cardiac surgery setting, peaks in $\mathrm{SCr}$ levels following $\mathrm{CPB}$ are not evident until 24-48 h after surgery. In a prospective observational study, Mamikonian et al. defined $\mathrm{AKI}$ as a doubling in $\mathrm{SCr}$ from preoperative baseline and using the pediatric-modified RIFLE criteria. Their results showed a significant increase in $\mathrm{SCr}$ as early as $2 \mathrm{~h}$ following completion of $\mathrm{CPB}$, with peak $\mathrm{SCr}$ levels occurring at $6 \mathrm{~h}$ after bypass [28].

\section{Factors influencing serum creatinine values Fluid overload}

In ICU patients, early and appropriate goal-directed fluid therapy is fundamental but is almost always associated with a certain degree of fluid overload responsible for tissue edema and progressive organ dysfunction. In this scenario, a positive fluid balance and lower urine volume are important factors associated with mortality of AKI patients [5, 29]. However, fluid management influences $\mathrm{SCr}$ and, using creatinine-based definitions, 'unrecognized' AKI could be identified after adjusting $\mathrm{SCr}$ concentration for positive fluid balance. A reanalysis of data from the Fluid and Catheter Treatment Trial demonstrated that correction of $\mathrm{SCr}$ for fluid balance unmasked the presence of AKI in some patients and led to a strong association between AKI and increased morbidity [6]. The adjustment of $\mathrm{SCr}$ concentration was performed using the following formula:

$$
\begin{aligned}
\text { Corrected } \mathrm{SCr}= & \text { measured } \mathrm{SCr} \\
& \times\left[1+\left(\frac{\text { accumulated net } \mathrm{FB}}{\mathrm{TBW}}\right)\right]
\end{aligned}
$$

where FB is fluid balance and TBW is total body water. The TBW is defined as: TBW $=0.6 \times$ weight $(\mathrm{kg})$.

Basu et al. analyzed the impact of this correction on pediatric AKI in a cardiac surgery setting using the same formula: the presence of AKI was associated with increased duration of mechanical ventilation, prolonged postoperative ICU length of stay, and hospital length of stay. In addition, patients with AKI had increased net fluid balance and required increased inotropic support [7]. In another study, Moore et al. [8] analyzed the effect of positive fluid balance on the incidence and the outcome of AKI in adult cardiac surgery patients. The authors adjusted the $\mathrm{SCr}$ concentration using the same formula, and then categorized patients into three groups based on the presence or absence of AKI using AKIN criteria and pre- and post- the adjustment: Group A, with no AKI pre or post-adjustment for fluid balance; Group B, with no AKI pre- but AKI post-adjustment for fluid balance; Group C, with AKI pre- and post-adjustment for fluid balance. The results showed that the adjustment of $\mathrm{SCr}$ for fluid balance was associated with outcomes that were midway between those of patients with and without AKI by standard criteria. This means that these patients may have less severe but still significant AKI but unfortunately have been incorrectly defined as AKI-free because of dilution of $\mathrm{SCr}$ [8].

\section{Muscle wasting}

$\mathrm{SCr}$ is a metabolite of creatine phosphate, an energy store found in skeletal muscle, and in normal subjects it is produced at a constant rate. A decrease in muscle mass could decrease SCr levels, and conversely SCr may be falsely increased with higher muscle mass [30]. $\mathrm{SCr}$ concentrations are extremely variable among elderly individuals and in children, as well as in critically ill patients. In this latter group of patients, muscle wasting starts already within the first week and is strongly related to poor outcome. Nevertheless, the early detection of muscle wasting is difficult. In a critical care setting, many conditions may lead to muscle wasting (e.g., malnourishment, immobilization, mechanical ventilation) [31], thus affecting SCr concentration, which may, therefore, appear to be within the reference range despite marked renal impairment. Consequently, the sensitivity of $\mathrm{SCr}$ for the early detection of AKI in these patients is poor. Fluid overload and malnutrition can seriously affect early creatinine-based AKI diagnosis and prognosis (Fig. 1). It is mandatory that the treating physician takes these factors into account when managing the

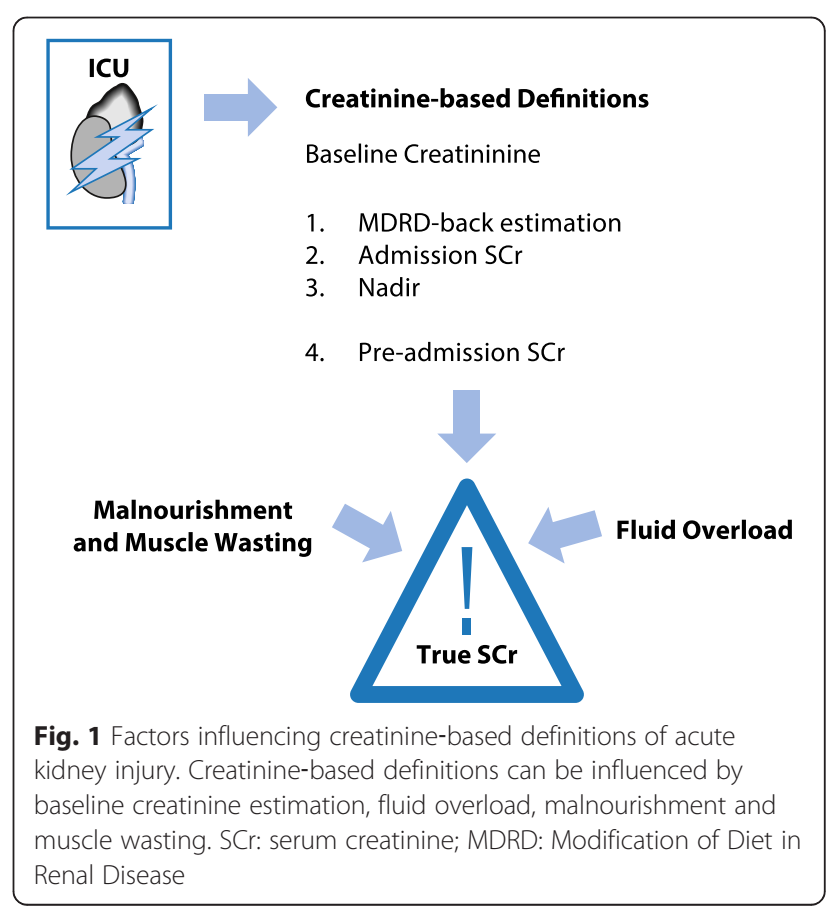


critically ill patient at risk of AKI. Future studies should address the effect of fluid overload and muscle wasting on the true $\mathrm{SCr}$ concentration.

\section{Susceptibility to kidney damage}

The diagnosis of subclinical AKI through the early identification of damage markers may lead to earlier initiation of AKI treatment or to adjustment of care, thus mitigating the adverse effects of AKI until renal function recovery. This approach could be relevant for risk stratification regarding future injury or subsequent CKD development. Emerging biomarkers have been identified that detect renal tubular damage, even in the absence of an increase in $\mathrm{SCr}$, and extend the spectrum of acute renal failure to early diagnostic evaluation and treatment of renal disorders [32]. In this scenario, the renal functional reserve (RFR) may be a relevant tool to predict the evolution of renal function. The RFR represents the capacity of intact nephron mass to increase GFR in response to stress and hence the difference between the peak stress $\mathrm{CrCl}$ induced by protein load and the baseline $\mathrm{CrCl}$ [33]. In physiological (e.g., pregnancy, solitary kidney) or pathological (e.g., diabetes mellitus, nephrotic syndrome) hyperfiltration states, the RFR allows the GFR to increase through mechanisms that are still unclear and under investigation. A recent theory suggests the presence of a population of "dormant cortical nephrons" uninvolved in filtration during resting conditions and potentially recruitable in response to stress; nevertheless these nephrons have never been demonstrated. Glomerular hyperfiltration, which may be considered another potential mechanism, is still controversial. Indeed, in most studies, the filtration fraction appears to be unaltered [34, 35], although some authors found an increase in the transcapillary hydraulic pressure gradient $[36,37]$. However, whether we consider the hypothetical recruitment of dormant cortical nephrons or the increase in net glomerular ultrafiltration pressure, these factors are both related to afferent vasodilation [34-36, 38], a mechanism which all authors agree on. Because pre-surgical kidney function is one of the most important determinants of both mortality and AKI outcomes [20, 39], it is reasonable to assume that the RFR plays a role in a patient's susceptibility to develop AKI.

\section{Conclusion}

The absence of a shared approach to AKI definitions has resulted in variability among centers, with different interpretations of $\mathrm{SCr}$ concentrations. The three possible methods used to define bSCr under- or over-estimate renal function in both cardiac surgery and ICU settings. In pediatrics, some authors have suggested the use of changes in eCrCl to define $\mathrm{AKI}$, and when the $\mathrm{bSCr}$ is unknown, use of the Schwartz $\mathrm{eCrCl}$ of $120 \mathrm{ml} / \mathrm{min}$ per $1.73 \mathrm{~m}^{2}$ could be useful to estimate baseline renal function. More studies and improved methods to estimate bSCr are clearly needed. Fluid overload and muscle wasting, often present in critically ill patients, can influence $\mathrm{SCr}$ and mask a diagnosis of AKI. Future investigations should address the estimation of true $\mathrm{SCr}$ values and the use of RFR as an important supportive tool for screening and prediction of kidney function recovery or progression to CKD.

\section{Competing interests \\ The authors declare that they have no competing interests.}

\section{Authors' contributions}

SD and SS have substantially contributed to the conception of the work, drafting the work or revising it critically for important intellectual content. CR has finally approved the version to be published. All authors read and approved the final manuscript.

\section{Declarations}

Publication of this article was funded by Associazione Amici del Rene di Vicenza (A.A.R.VI. onlus).

Published online: 15 March 2016

References

1. Koyner JL. Assessment and diagnosis of renal dysfunction in the ICU. Chest. 2012;141:1584-94.

2. Roy AK, Mc Gorrian C, Treacy C, et al. A comparison of traditional and novel definitions (RIFLE, AKIN, and KDIGO) of acute kidney injury for the prediction of outcomes in acute decompensated heart failure. Cardiorenal Med. 2013;3:26-37.

3. Bagshaw SM, Uchino S, Cruz D, et al. A comparison of observed versus estimated baseline creatinine for determination of RIFLE class in patients with acute kidney injury. Nephrol Dial Transplant. 2009;24:2739-44.

4. Lafrance JP, Miller DR. Defining acute kidney injury in database studies: the effects of varying the baseline kidney function assessment period and considering CKD status. Am J Kidney Dis. 2010;56:651-60.

5. Teixeira C, Garzotto F, Piccinni P, et al. Fluid balance and urine volume are independent predictors of mortality in acute kidney injury. Crit Care. 2013;17:R14.

6. Liu KD, Thompson BT, Ancukiewicz M, et al. Acute kidney injury in patients with acute lung injury: impact of fluid accumulation on classification of acute kidney injury and associated outcomes. Crit Care Med. 2011;39:2665-71.

7. Basu RK, Andrews A, Krawczeski C, et al. Acute kidney injury based on corrected serum creatinine is associated with increased morbidity in children following the arterial switch operation. Pediatr Crit Care Med. 2013;14:e218-24.

8. Moore E, Tobin A, Reid D, et al. The impact of fluid balance on the detection, classification and outcome of acute kidney injury after cardiac surgery. J Cardiothorac Vasc Anesth. 2015;29:1229-35.

9. Puthucheary ZA, Rawal J, McPhail M, et al. Acute skeletal muscle wasting in critical illness. JAMA. 2013;310:1591-600.

10. Larsson L. Acute quadriplegic myopathy: an acquired "myosinopathy". Adv Exp Med Biol. 2008;642:92-8.

11. Bellomo R, Ronco C, Kellum JA, Mehta RL, Palevsky P. Acute renal failure - definition, outcome measures, animal models, fluid therapy and information technology needs: the Second International Consensus Conference of the Acute Dialysis Quality Initiative (ADQI) Group. Crit Care. 2004;8:R204-12.

12. Bagshaw SM, George C, Bellomo R. A comparison of the RIFLE and AKIN criteria for acute kidney injury in critically ill patients. Nephrol Dial Transplant. 2008:23:1569-74.

13. Joannidis $M$, Metnitz $B$, Bauer $P$, et al. Acute kidney injury in critically ill patients classified by AKIN versus RIFLE using the SAPS 3 database. Intensive Care Med. 2009;35:1692-702. 
14. Siew ED, Ikizler TA, Matheny ME, et al. Estimating baseline kidney function in hospitalized patients with impaired kidney function. Clin J Am Soc Nephrol. 2012;7:712-9.

15. Siew ED, Matheny ME, Ikizler TA, et al. Commonly used surrogates for baseline renal function affect the classification and prognosis of acute kidney injury. Kidney Int. 2010;77:536-42.

16. Broce JC, Price LL, Liangos O, Uhlig K, Jaber BL. Hospital-acquired acute kidney injury: an analysis of nadir-to-peak serum creatinine increments stratified by baseline estimated GFR. Clin J Am Soc Nephrol. 2011;6:1556-65.

17. Matheny ME, Peterson JF, Eden SK, et al. Laboratory test surveillance following acute kidney injury. PLoS One. 2014;9:e103746.

18. Rosner MH, Okusa MD. Acute kidney injury associated with cardiac surgery. Clin J Am Soc Nephrol. 2006;1:19-32.

19. Nashef SA, Roques F, Sharples LD, et al. EuroSCORE II. Eur J Cardiothorac Surg. 2012;41:734-44.

20. Wijeysundera DN, Karkouti K, Dupuis JY, et al. Derivation and validation of a simplified predictive index for renal replacement therapy after cardiac surgery. JAMA. 2007;297:1801-9.

21. Chertow GM, Levy EM, Hammermeister KE, Grover F, Daley J. Independent association between acute renal failure and mortality following cardiac surgery. Am J Med. 1998;104:343-8.

22. Mangano CM, Diamondstone LS, Ramsay JG, Aggarwal A, Herskowitz A, Mangano DT. Renal dysfunction after myocardial revascularization: risk factors, adverse outcomes, and hospital resource utilization. The Multicenter Study of Perioperative Ischemia Research Group. Ann Intern Med. 1998;128:194-203.

23. Wagener $G$, Jan M, Kim M, et al. Association between increases in urinary neutrophil gelatinase-associated lipocalin and acute renal dysfunction after adult cardiac surgery. Anesthesiology. 2006;105:485-91.

24. Meersch M, Schmidt C, Van Aken H, et al. Urinary TIMP-2 and IGFBP7 as early biomarkers of acute kidney injury and renal recovery following cardiac surgery. PLoS One. 2014;9:e93460.

25. Candela-Toha AM, Recio-Vazquez M, Delgado-Montero A, et al. The calculation of baseline serum creatinine overestimates the diagnosis of acute kidney injury in patients undergoing cardiac surgery. Nefrologia. 2012;32:53-8.

26. Zappitelli M, Parikh CR, Akcan-Arikan A, Washburn KK, Moffett BS, Goldstein SL. Ascertainment and epidemiology of acute kidney injury varies with definition interpretation. Clin J Am Soc Nephrol. 2008;3:948-54.

27. Alkandari $\mathrm{O}$, Eddington KA, Hyder $\mathrm{A}$, et al. Acute kidney injury is an independent risk factor for pediatric intensive care unit mortality, longer length of stay and prolonged mechanical ventilation in critically ill children: a two-center retrospective cohort study. Crit Care. 2011;15:R146.

28. Mamikonian LS, Mamo LB, Smith PB, Koo J, Lodge AJ, Turi JL. Cardiopulmonary bypass is associated with hemolysis and acute kidney injury in neonates, infants, and children. Pediatr Crit Care Med. 2014;15:e111-9.

29. Bouchard J, Soroko SB, Chertow GM, et al. Fluid accumulation, survival and recovery of kidney function in critically ill patients with acute kidney injury. Kidney Int. 2009;76:422-7.

30. Baxmann AC, Ahmed MS, Marques NC, et al. Influence of muscle mass and physical activity on serum and urinary creatinine and serum cystatin C. Clin J Am Soc Nephrol. 2008:3:348-54.

31. Lodeserto F, Yende S. Understanding skeletal muscle wasting in critically ill patients. Crit Care. 2014;18:617.

32. Haase M, Kellum JA, Ronco C. Subclinical AKI - an emerging syndrome with important consequences. Nat Rev Nephrol. 2012;8:735-9.

33. Sharma A, Mucino MJ, Ronco C. Renal functional reserve and renal recovery after acute kidney injury. Nephron Clin Pract. 2014;127:94-100.

34. Hostetter TH. Human renal response to meat meal. Am J Physiol. 1986;250:F613-8.

35. Solling K, Christensen CK, Solling J, Christiansen JS, Mogensen CE. Effect on renal haemodynamics, glomerular filtration rate and albumin excretion of high oral protein load. Scand J Clin Lab Invest. 1986;46:351-7.

36. Chan AY, Cheng ML, Keil LC, Myers BD. Functional response of healthy and diseased glomeruli to a large, protein-rich meal. J Clin Invest. 1988;81:245-54.

37. Rodriguez-Iturbe B, Herrera J, Garcia R. Relationship between glomerular filtration rate and renal blood flow at different levels of protein-induced hyperfiltration in man. Clin Sci (Lond). 1988;74:11-5.

38. ter Wee PM, Geerlings W, Rosman JB, et al. Testing renal reserve filtration capacity with an amino acid solution. Nephron. 1985;41:193-9.

39. Thakar CV, Arrigain S, Worley S, Yared JP, Paganini EP. A clinical score to predict acute renal failure after cardiac surgery. J Am Soc Nephrol. 2005;16:162-8. 